\title{
The association analysis of hOGG1 genetic variants and gastric cancer risk in a Chinese population
}

\author{
Jiafei Lu ${ }^{1,2, *}$, Yongmei Yin ${ }^{3, *}$, Mulong Du ${ }^{1,2, *}$, Gaoxiang Ma ${ }^{1,2}$, Yuqiu Ge ${ }^{1,2}$, Qiang \\ Zhang ${ }^{1,2}$, Haiyan Chu ${ }^{1,2}$, Na Tong ${ }^{1,2}$, Meilin Wang ${ }^{1,2}$, Jinrong Qiu ${ }^{3,4}$, Zhengdong \\ Zhang ${ }^{1,2}$ \\ ${ }^{1}$ Department of Environmental Genomics, Jiangsu Key Laboratory of Cancer Biomarkers, Prevention and Treatment, \\ Collaborative Innovation Center for Cancer Personalized Medicine, Nanjing Medical University, Nanjing, China \\ ${ }^{2}$ Department of Genetic Toxicology, The Key Laboratory of Modern Toxicology of Ministry of Education, School of Public \\ Health, Nanjing Medical University, Nanjing, China \\ ${ }^{3}$ Department of Oncology, The First Affiliated Hospital of Nanjing Medical University, Nanjing, China \\ ${ }^{4}$ Department of Biological-Therapy, Eastern Hepatobiliary Surgery Hospital Affiliated to Second Military Medical University, \\ Shanghai, China \\ *These authors have contributed equally to this work \\ Correspondence to: Zhengdong Zhang, email: drzdzhang@gmail.com \\ Jinrong Qiu, email: jrqiu@njmu.edu.cn \\ Keywords: polymorphism, hOGG 1, gastric cancer, susceptibility
}

Received: March 22, $2016 \quad$ Accepted: August 24, 2016

Published: September 01, 2016

\section{ABSTRACT}

Human 8-oxoguanine DNA glycosylase (hOGG1) is known to play an important role in the prevention of carcinogenesis, including gastric cancer (GC). We performed a case-control study to investigate whether single nucleotide polymorphisms (SNPS) of hOGG1 are associated with GC risk in a Chinese population. Two potential functional tagSNPs (rs159153 and rs1052133) and a previously reported risk SNP (rs125701) were genotyped in 1,275 GC patients and 1,436 controls. We found that SNP rs125701 G > A was significantly associated with the increased GC risk [adjusted odds ratio $(O R)=1.38,95 \%$ confidence interval $(C I)=1.05-1.79$ in additive model $]$. Besides, the functional studies demonstrated that the minor A allele of rs125701 significantly reduced the transcriptional activity of hOGG1 promoter and enhanced the methylation level of CpG site of cg15357639. In conclusion, our results suggested that the SNP rs125701 in hOGG1 promoter was associated with the elevated GC risk, which could act as a new potential biomarker for GC susceptibility. Further functional verification of rs125701 in GC pathogenesis is warranted.

\section{INTRODUCTION}

Gastric cancer is the fifth most common malignancy worldwide, with an estimated 951,600 new cases and 723,100 deaths occurring in 2012 [1]. Although the decline trends in the GC morbidity have been noted in recent years, it remains the second leading cause of cancer deaths in China, with probably 498,000 deaths in 2015 [2]. The overall 5-year survival rate of GC is still poor, as GC patients are mostly diagnosed at advanced stage, during which any treatment is unreliable. Therefore, the identification and control of risk factors might be useful for reducing the prevalence of GC [3-5]. It is wellknown that the pathogenetic mechanism of GC is very complicated. Accumulating evidence has proved that there exist a correlation between genetic polymorphisms and GC risk [6-8].

DNA damage is involved in carcinogenesis [9]. As a key component of DNA repair pathway, $h O G G 1$ encodes a DNA glycosylase specifically involved in the repair of DNA oxidative damage [10,11]. The dysfunction of hOGG1 might cause the DNA repair deficiency and then induce gene mutation and cell canceration. Abnormal expression of hOGG1 was detected in several tumor tissues, such as ovarian cancer [12], breast cancer [13], and gastric cancer [14]. In addition, studies indicated that esophageal squamous cell carcinoma (ESCC) cells transfected with vector containing $h O G G 1$ could exhibit 
lower cell apoptosis, less oxidative damage, and longer survival ability compared with no-treated ESCC cells [15].

SNPs in DNA repair genes may conclusively affect individual variation in DNA repair capability and modulate individual cancer susceptibility. So investigation of SNPs in $h O G G 1$ contributes to uncovering pathogenesis of GC. Currently, the most frequently studied SNP of hOGG1 was Ser326Cys polymorphism (rs1052133), still having inconsistent results with GC susceptibility [16-19]. In our previous study, we have conducted a meta-analysis to prove that the Ser326Cys polymorphism is significantly associated with an elevated risk of GC [20]. However, few studies have given attention to other $h O G G 1$ polymorphisms and GC susceptibility. In view of the importance of $h O G G 1$ in tumorigenesis, we thought that the roles of other SNPs in hOGG1 deserve to be explored as well. Thus, in this study, we screened potential functional tagSNPs in $h O G G 1$ and its upstream $2000 \mathrm{bp}$ region to explore the association between the SNPs in hOGG1 and GC risk. In addition, SNPs in $h O G G 1$ that have been previously reported to be associated with cancers were recruited into our study.

\section{RESULTS}

\section{Characteristics of study subjects}

In this study, we found no significant difference among cases and controls in the distributions of age $(P=0.595)$ and sex $(P=0.349)$. Clinicopathological characteristics of GC patients are summarized in Supplementary Table S1. Of the cases, $61.3 \%$ of them were in non-cardia type, and $33.6 \%$ in the cardia and $5.1 \%$ in the both. In addition, $682(61.4 \%)$ had lymph node metastasis and $167(15.1 \%)$ existed distant metastasis. According to the TNM classification, all the cases were identified to stage I, II, III, and IV with the percentage of $23.1 \%, 24.6 \%, 35.5 \%$, and $16.8 \%$, respectively.

\section{Association analysis between the selected SNPs in $h O G G 1$ and $\mathrm{GC}$ risk}

The position of three selected SNPs in hOGGl was shown in Figure 1A. The genotype distribution of the selected SNPs and their associations with GC risk are shown in Supplementary Table S2. The genotype frequencies of all SNPs among the controls were consistent with HardyWeinberg equilibrium $(P=0.260$ for rs 1052133, $P=0.125$ for rs159153 and $P=0.518$ for rs125701). We found that only SNP rs 125701 showed significant difference between the cases and controls ( $P=0.018$ in adjusted additive model).

Subsequently, various inheritance models were used to determine the associations of rs $125701 \mathrm{G}>\mathrm{A}$ polymorphism and GC risk (Table 1). Results of additive model indicated that the genotype distribution of rs125701 was significantly different between the cases and controls (adjusted $\mathrm{OR}=1.38$, $95 \% \mathrm{CI}=1.05-1.79, P=0.018)$. Compared with the major
GG genotype in a codominant model, the variant A alleles correlated to an increased risk of GC, showing a distinct allele-dosage effect $\left(P_{\text {trend }}=0.022\right)$. We found that SNP rs125701 also had risk effects on GC risk in the dominant model (adjusted $\mathrm{OR}=1.33,95 \% \mathrm{CI}=1.00-1.75, P=0.047$ ) and recessive model (adjusted $\mathrm{OR}=9.14,95 \% \mathrm{CI}=1.14$ $73.06, P=0.037)$. Taken together, the rs125701 A allele was considered to be a potential risk allele for GC.

\section{Stratified analysis of SNP rs125701 and GC risk}

We further evaluated the effects of rs125701 polymorphism on GC risk stratified according to demographic and clinicopathological characteristics. We did not find any association between rs125701 genotypes and GC susceptibility in subgroups of different age or sex (Supplementary Table S3). However, significant risk effect of rs 125701 AG/AA genotype was observed among patients with non-cardia (adjusted OR $=1.53,95 \% \mathrm{CI}=$ $1.12-2.09$ ), histological types of diffuse (adjusted $\mathrm{OR}=$ $1.43,95 \% \mathrm{CI}=1.02-1.99)$ and lymph node metastasis $($ adjusted OR $=1.44,95 \% \mathrm{CI}=1.04-1.99)($ Table 2$)$.

\section{Effects of SNP rs125701 on transcriptional activity}

To examine the biological effect of SNP rs125701 on the $h O G G 1$ promoter, different recombinant plasmids containing the $h O G G 1$ promoter region with rs $125701 \mathrm{~A}$ or G allele (pGL3-GG/AA, Figure 1B) were transfected into MGC-803 and BGC-823 GC cells respectively. Then the relative transcriptional activity was evaluated via measuring firefly and renilla fluorescent intensity. As shown in Figure 1C, the luciferase activity of the vectors with rs125701 A allele was significantly decreased compared to that of $\mathrm{G}$ allele in both above cells $(P=0.021$ and 0.002 , respectively). These results indicated that rs 125701 significantly affected the luciferase gene expression in vitro.

\section{In silico analysis for the allele-specific effect of rs125701}

Next, the effect of SNP rs125701 on hOGG1 expression was explored in the expression quantitative trait locus (eQTL) analysis through The Cancer Genome Atlas (TCGA) database. Regrettably, we did not discover any statistical difference of the $h O G G 1$ mRNA levels between different rs125701 genotypes from both GC patients and cancer-free controls $(P=0.541$ for cases and 0.317 for controls) (Supplementary Figure S1).

We also tested the $\mathrm{CpG}$ sites methylation status situated near the SNP rs 125701 through UCSC database. Interestingly, we found a $\mathrm{CpG}$ site of $\operatorname{cg} 15357639$ with high methylation activity located 34 bases upstream of SNP rs125701 (Figure 1A). Thus we speculated whether the rs125701 polymorphism correlates to the methylation 
Table 1: Association of $h O G G 1$ rs125701 polymorphism with gastric cancer risk

\begin{tabular}{|c|c|c|c|c|c|c|}
\hline \multirow[t]{2}{*}{ Genotype } & \multicolumn{2}{|c|}{ cases } & \multicolumn{2}{|c|}{ controls } & \multirow[t]{2}{*}{ Adjusted OR(95\%CI) } & \multirow[t]{2}{*}{$P^{\mathrm{a}}$} \\
\hline & $\mathbf{N}$ & $\%$ & $\mathbf{N}$ & $\%$ & & \\
\hline \multicolumn{7}{|c|}{ Codominant model } \\
\hline GG & 1159 & 90.9 & 1334 & 92.9 & $1.00($ reference $)$ & \\
\hline $\mathrm{AG}$ & 108 & 8.5 & 101 & 7.0 & $1.25(0.94,1.65)$ & 0.127 \\
\hline AA & 8 & 0.6 & 1 & 0.1 & $9.29(1.16,74.23)$ & 0.036 \\
\hline$P$ trend & & & & & & 0.022 \\
\hline Additive model & & & & & $1.38(1.05,1.79)$ & 0.018 \\
\hline \multicolumn{7}{|l|}{ Dominant model } \\
\hline GG & 1159 & 90.9 & 1334 & 92.9 & $1.00($ reference $)$ & \\
\hline $\mathrm{AG} / \mathrm{AA}$ & 116 & 9.1 & 102 & 7.1 & 1.33(1.00,1.75) & 0.047 \\
\hline \multicolumn{7}{|l|}{ Recessive model } \\
\hline $\mathrm{GG} / \mathrm{AG}$ & 1276 & 99.4 & 1435 & 99.9 & $1.00($ reference $)$ & \\
\hline AA & 8 & 0.6 & 1 & 0.1 & $9.14(1.14,73.06)$ & 0.037 \\
\hline \multicolumn{7}{|l|}{ Allele $^{b}$} \\
\hline G & 2426 & 95.1 & 2769 & 96.4 & $1.00($ reference $)$ & \\
\hline A & 124 & 4.9 & 103 & 3.6 & $1.37(1.05,1.80)$ & 0.019 \\
\hline
\end{tabular}

a Adjusted by age, sex in logistic regression analysis

b Two-sided $\chi^{2}$ test for allele frequencies between the cases and controls

level of the $\mathrm{CpG}$ site and the methylation quantitative trait locus (meQTL) analysis was performed. As shown in Figure 2, prominent trend among the three genotype groups was existent: the more A allele, the higher methlation level of cg15357639 was found in GC patients $\left(P_{\text {trend }}=0.016\right)$.

\section{DISCUSSION}

The hOGGl gene is located on chromosome 3 p25-26, in which a frequent loss of heterozygosity was observed during tumorigenesis [21, 22]. Mice lacking hOGG1 exhibit higher incidence of lung carcinogenesis and insulin resistance [23]. The abnormal expression of $h O G G 1$ was detected in several tumors $[12,13,15]$ including GC [14]. In addition, the SNP rs1052133 in hOGG1 and cancer susceptibility has been identified in numerous tumors including esophageal cancer [24], prostate cancer [25], lung cancer [26], breast cancer [27] and gastric cancer [28] and so on. All of these indicated important biological role of $h O G G 1$ in cancer etiology.

In this study, we conducted a case-control study to estimate the association between three candidate SNPs in $h O G G 1$ and GC risk. We found that individuals carrying the rs125701 AG/AA genotypes had a significantly increased GC risk, compared with those with the GG genotype. The SNP rs125701 was previously identified to have a suggestive association with high-grade prostate cancer in the US research [29] and a significantly reduced risk to bladder cancer from the Spanish Bladder Cancer Study (SBCS) [30]. In addition, the risk effect of rs 125701 was more prominent among patients with non-cardia cancer than that with cardia cancer. Gastric cardia cancer differs from non-cardia cancer in anatomic site, etiology and clinical characteristics. Accumulating evidence has proved that many SNPs show different associations between the two main subsites of GC. SNP rs2294693 near $U N C 5 C L$ at $6 \mathrm{p} 21.1$ [31] and SNP rs9841504 in ZBTB20 [32] was reported to be associated with gastric non-cardia cancer. SNP rs2274223 located in PLCE1 was associated with cardia but not non-cardia GC [33]. These findings that identified phenotype-specific genetic susceptibility loci may contribute to understanding the etiology and mechanisms of different subtypes of GC. The data published to date about GC risk and rs 1052133 polymorphism is inconsistent. In our study, the SNP rs1052133 did not show a significant correlation to GC risk. This difference in susceptibility of rs 1052133 to GC might be due to the different size of study sample or the gene-environment interactions and genetic background in different populations.

Currently, no functional study was performed to estimate the role of rs125701 polymorphism in the etiology of malignancy. In this study, luciferase reporter 
Table 2: Associations between rs125701 genotypes and clinical characteristics of GC

\begin{tabular}{|c|c|c|c|c|c|c|}
\hline \multirow[t]{2}{*}{ Variables } & \multicolumn{2}{|c|}{ GG } & \multicolumn{2}{|c|}{ AG/AA } & \multirow[t]{2}{*}{ Adjusted OR (95\% CI) } & \multirow[t]{2}{*}{$P^{\mathrm{a}}$} \\
\hline & $\mathbf{N}$ & $\%$ & $\mathbf{N}$ & $\%$ & & \\
\hline Controls & 1334 & 92.90 & 102 & 7.10 & $1.00($ reference $)$ & \\
\hline \multicolumn{7}{|l|}{ Tumor site } \\
\hline Cardia & 373 & 92.56 & 30 & 7.44 & $1.08(0.70,1.65)$ & 0.734 \\
\hline Non-cardia & 658 & 89.65 & 76 & 10.35 & $1.53(1.12,2.09)$ & 0.008 \\
\hline \multicolumn{7}{|l|}{ Histological types } \\
\hline Diffuse & 552 & 90.20 & 60 & 9.80 & 1.43(1.02,1.99) & $\mathbf{0 . 0 3 8}$ \\
\hline Intestinal & 470 & 91.62 & 43 & 8.38 & $1.22(0.94,1.77)$ & 0.303 \\
\hline \multicolumn{7}{|l|}{ Depth of invasion } \\
\hline $\mathrm{T} 1$ & 157 & 92.35 & 13 & 7.65 & $1.08(0.59,1.97)$ & 0.798 \\
\hline $\mathrm{T} 2$ & 151 & 89.35 & 18 & 10.65 & $1.59(0.99,2.69)$ & 0.088 \\
\hline $\mathrm{T} 3$ & 521 & 90.61 & 54 & 9.39 & $1.37(0.97,1.93)$ & 0.075 \\
\hline $\mathrm{T} 4$ & 187 & 92.57 & 15 & 7.43 & $1.05(0.60,1.85)$ & 0.856 \\
\hline \multicolumn{7}{|l|}{ Lymph node metastasis } \\
\hline No & 397 & 92.76 & 31 & 7.24 & $1.03(0.68,1.56)$ & 0.893 \\
\hline $\mathrm{N} 1 / \mathrm{N} 2 / \mathrm{N} 3$ & 615 & 90.18 & 67 & 9.82 & 1.44(1.04,1.99) & 0.028 \\
\hline \multicolumn{7}{|l|}{ Distant metastasis } \\
\hline M0 & 858 & 91.18 & 83 & 8.82 & $1.27(0.94,1.71)$ & 0.127 \\
\hline M1 & 154 & 92.22 & 13 & 7.78 & $1.14(0.63,2.09)$ & 0.662 \\
\hline \multicolumn{7}{|l|}{ TNM stages } \\
\hline Localized (I+II) & 499 & 90.89 & 50 & 9.11 & $1.33(0.93,1.90)$ & 0.114 \\
\hline Advanced(III+IV) & 550 & 91.06 & 54 & 8.94 & $1.30(0.92,1.83)$ & 0.142 \\
\hline
\end{tabular}

${ }^{a}$ Adjusted by age and sex in logistic regression analysis
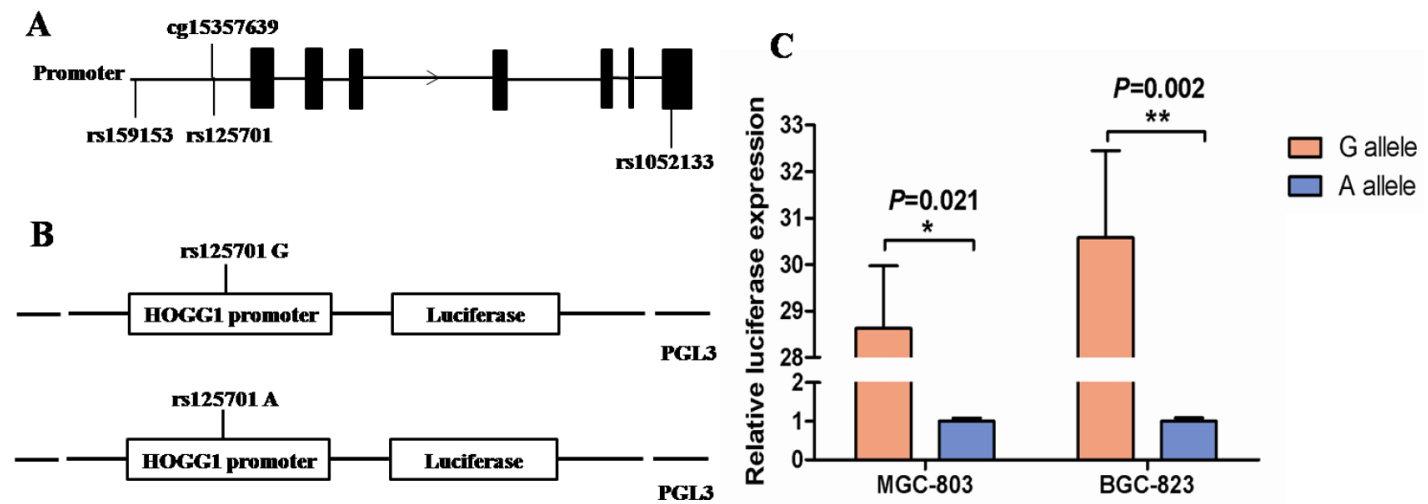

Figure 1: Effect of rs125701 polymorphism in the $\boldsymbol{h O G G 1}$ promoter activity. A. Relative position of selected three SNPs and a CpG site of cg15357639 in hOGG1 gene. B. Schematic representation of reporter plasmids containing the rs125701 G or A allele, which was inserted upstream of the luciferase reporter gene in the pGL3 basic plasmid. C. The transcriptional activity of the constructs with rs 125701 A allele was significantly lower than that of G allele in both MGC-803 and BGC-823 cells $(P=0.021$ and 0.002 , respectively). Columns represent mean from three independent experiments and bars means SD. 


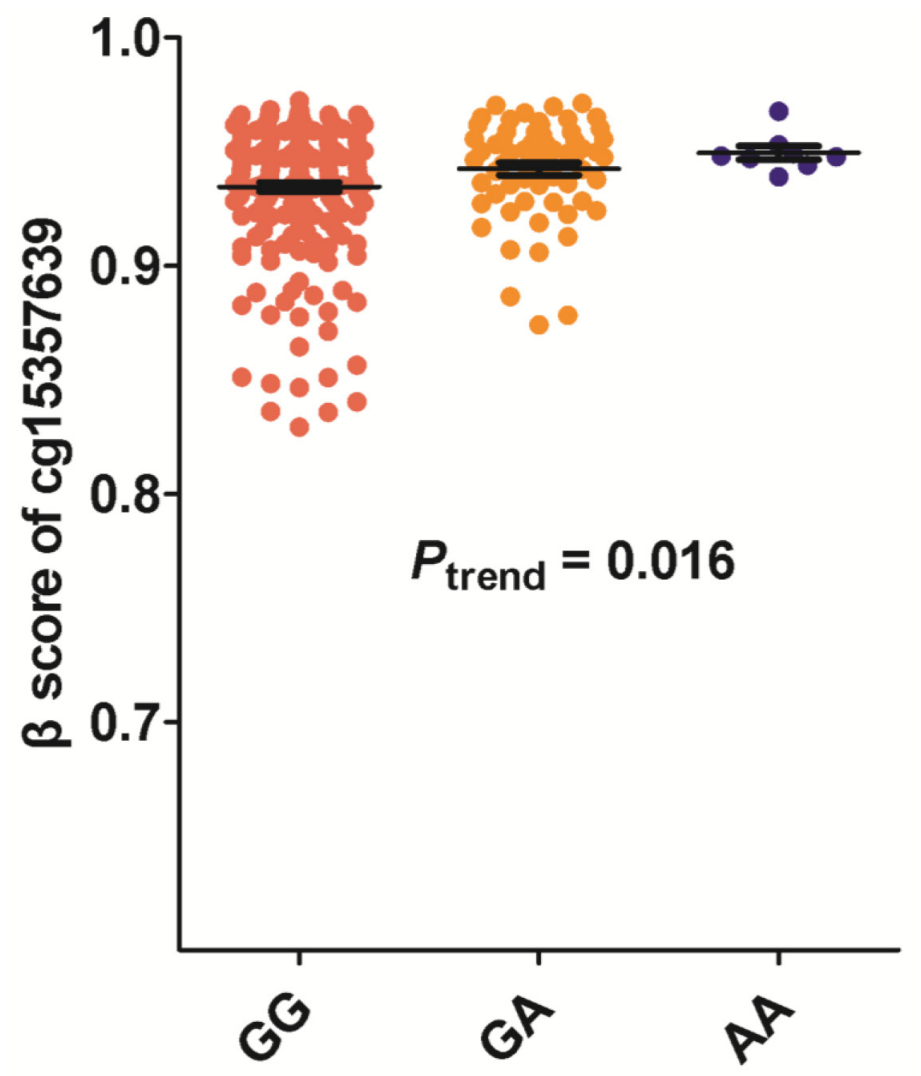

Figure 2: Association between rs125701 polymorphism and the methylation level of CpG site of cg15357639. There was prominent trend of increased methylation activity of $\mathrm{CpG}$ site of $\operatorname{cg} 15357639$ among the three genotype groups $\left(P_{\text {trend }}=0.016\right)$.

gene assay indicated that rs125701 A allele dramatically reduced the transcription activity of $h O G G 1$ promoter; and the rs125701 A allele was associated with elevated methylation level of $\mathrm{CpG}$ site of $\operatorname{cg} 15357639$ in the meQTL analysis. The rs125701 polymorphism is located adjacent to the $\mathrm{CpG}$ site of $\operatorname{cg} 15357639$, which is able to undergo methylation. Previous studies have demonstrated that DNA methylation is associated with SNPs, which may modify the $\mathrm{CpG}$ sites methylation or influence the generation of new CpG sites, change the status of genes' methylation and contribute to tumorigenesis in turn [3436]. Furthermore, Julietet al. found that functional risk SNPs (rs554219 and rs78540526) at the 11q13 locus for breast cancer could regulate $C C N D 1$ expression through long-range regulation, which located approximately $125 \mathrm{~kb}$ downstream [37]. Taken together, we speculated that the SNP rs125701 could suppress the expression level of other nearby genes instead of $h O G G 1$ by decreasing transcription activity or enhancing methylation level of the region where this SNP is located.

Several limitations of our study should be mentioned. First, as vital roles in gastric carcinogenesis, the smoking, drinking and Helicobacter pylori infection information were devoid in our study. The significance of rs125701 should be further validated to investigate the gene-environment interaction in the pathogenesis of
GC. Second, the SNP rs125701 in additive model was of marginal difference between cases and controls after the Bonferroni correction $(P=0.054)$. The weak association between SNP rs125701 and GC risk might due to the small sample size in our study. This result should be verified by increasing the sample size or performing another casecontrol study in an independent population. Third, another six tagSNPs we identified in intron of $h O G G 1$ were neglected in our study, which may also be significant and their effect on GC risk should be evaluated.

In summary, we demonstrated that SNP rs125701 of $h O G G 1$ was associated with increased GC risk in the Chinese populations. Meanwhile, the polymorphism rs125701 leads to reduced transcription activity and enhanced methylation level of the promoter region, which might inhibit the expression of other nearby genes instead of $h O G G 1$. These findings should be verified by larger, well-designed epidemiologic and functional studies.

\section{MATERIALS AND METHODS}

\section{Study subjects}

There were 1,275 GC cases and 1,436 cancerfree controls included in our study. All cases were histopathologically confirmed as gastric adenocarcinoma 
and recruited from the Cancer Clinical Research Base of Nanjing Medical University between March 2006 and May 2013. The control subjects were randomly enrolled at the same period when they sought physical examinations at hospital. In addition, the finally selected controls were frequency-matched to cases on age ( \pm 5 years) and sex. All eligible but only genetically unrelated ethnic Han Chinese patients were remained in this study. We acquired demographic and clinical information for all the subjects after a written informed consent was signed. A $5 \mathrm{~mL}$ peripheral venous blood sample was donated from each individual after the interview. The study was approved by the institutional review board of Nanjing Medical University.

\section{SNPs selection}

SNPs located in $h O G G 1$ and its upstream 2000 bps region were searched based on genotype data of Asian population from the 1000 Genomes Project. We identified eight tagSNPs covering all the common SNPs (minor allele frequency, MAF > 0.05) using the Haploview 4.2 software (Cambridge, MA, USA) with a standard of $r^{2}$ at least 0.8. We would like to focus on SNPs in functional region, including the region of promoter, 5' -untranslated region (5'-UTR), and exon. Among the 8 tagSNPs, 2 of them are located in promoter (rs159153) and exon (rs 1052133). Another 6 tagSNPs and their highly linked loci are all located in intron of $h O G G 1$ (Supplementary Table S4). Besides, SNPs that have been previously reported to be involved in cancer were recruited in our study. Finally, three SNPs (rs159153, rs1052133 and rs125701) remained in further analysis (Figure 1A).

\section{Genotyping}

Genomic DNA was extracted from peripheral blood of each study subject. TaqMan allelic discrimination assay was used to genotype the selected SNPs by using the ABI 7900HT Real-Time PCR System (Applied Biosystems, Foster City, CA, USA). Sequences of primers and probes are summarized in Supplementary Table S5.

The average call rate of the three SNPs reached $99 \%$. To control the quality, we randomly selected $10 \%$ of the samples to genotype again, and the results were $100 \%$ concordant.

\section{Construction of promoter reporter plasmids}

As shown in Figure 1B, the $h O G G 1$ promoter region containing SNP rs125701 G or A allele was synthesized and then inserted into a pGL3-basic luciferase reporter plasmid (Promega, Madison, WI, USA) using the NheI and XhoI enzymes. The recombinant plasmids were sequenced to confirm the orientation and integrity of each construct.

\section{Transfection and luciferase assay}

The luciferase reporter assay was used to detect the effect of SNP rs125701 on $h O G G 1$ promoter activity. MGC-803 and BGC-823 cells were cultured in 24well plates and transfected with $800 \mathrm{ng}$ of recombinant plasmids using Lipofectamine ${ }^{\mathrm{TM}} 2000$ (Invitrogen, Carlsbad, CA, USA). The 10 ng Renilla luciferase pRLSV40 (Promega, Madison, WI, USA) was simultaneously cotransfected into cells per well as internal control. After 24 hours transfection, the cells were lysed and measured by the Dual-Luciferase Reporter Assay System (Promega, Madison, WI, USA). Relative luciferase activity was estimated by the ratio of Firefly and Renilla fluorescent intensity. Each transfection was carried out in independent triplicate.

\section{Statistical analysis}

The data were analyzed using SAS software (version 9.1.3; SAS Institute, Cary, NC). Student's $t$ test and Pearson's chi-squared $\left(\chi^{2}\right)$ test were used to assess the differences in demographic factors between cases and controls. Hardy-Weinberg equilibrium (HWE) of the controls was done by a goodness-of-fit $\chi^{2}$ test. The ORs and $95 \%$ CIs were calculated by an unconditional logistic regression model for the associations between genotype distribution and GC susceptibility. Variables of age and sex were as covariates adjusted for the association analysis. Multiple inheritance models were applied to estimate the significance of SNP rs125701. The promoter activity was analyzed by a Student's $t$ test. $P<0.05$ for two-sided was considered statistically significant.

\section{ACKNOWLEDGMENTS}

This study was partly supported by National Natural Science Foundation of China (81301690 and 81230068), the Key Program for Basic Research of Jiangsu Provincial Department of Education (12KJA330002), Jiangsu Provincial Science and Technology Innovation Team, Collaborative Innovation Center for Cancer Personalized Medicine, and the Priority Academic Program Development of Jiangsu Higher Education Institutions (Public Health and Preventive Medicine).

\section{CONFLICTS OF INTEREST}

We declared no conflicts of interests in this study.

\section{REFERENCES}

1. Torre LA, Bray F, Siegel RL, Ferlay J, Lortet-Tieulent J and Jemal A. Global cancer statistics, 2012. CA Cancer J Clin. 2015; 65:87-108. 
2. Chen W, Zheng R, Baade PD, Zhang S, Zeng H, Bray F, Jemal A, Yu XQ and He J. Cancer statistics in China, 2015. CA Cancer J Clin. 2016.

3. Dicken BJ, Bigam DL, Cass C, Mackey JR, Joy AA and Hamilton SM. Gastric adenocarcinoma: review and considerations for future directions. Ann Surg. 2005; 241:27-39.

4. Khan FA and Shukla AN. Pathogenesis and treatment of gastric carcinoma: "an up-date with brief review". J Cancer Res Ther. 2006; 2:196-199.

5. Catalano V, Labianca R, Beretta GD, Gatta G, de Braud F and Van Cutsem E. Gastric cancer. Crit Rev Oncol Hematol. 2009; 71:127-164.

6. Gu H, Yang L, Tang N, Zhou B, Zhu H, Sun Q, Cong R and Wang B. Association of endothelin-converting enzyme- $1 \mathrm{~b}$ C-338A polymorphism with gastric cancer risk: a casecontrol study. Eur J Cancer. 2008; 44:1253-1258.

7. Yang L, Liu D, Liang S, Guo R, Zhang Z, Xu H, Yang C and Zhu Y. Janus kinase 2 polymorphisms are associated with risk in patients with gastric cancer in a Chinese population. PLoS One. 2013; 8:e64628.

8. Qian F, Sun BL, Zhang WY, Ke J and Zhu J. Gly82Ser polymorphism of the receptor for advanced glycation end-product (RAGE) potential high risk in patients with colorectal cancer. Tumour Biol. 2014; 35:3171-3175.

9. Christmann M, Tomicic MT, Roos WP and Kaina B. Mechanisms of human DNA repair: an update. Toxicology. 2003; 193:3-34

10. Liu X, Xiao N, Guo W, Wu Y, Cai Z, He Q, Zhang L, Chen X, Sun C, Wang J, Zhu C, Jin H and Wang Y. The hOGG1 gene 5'-UTR variant c.-53G $>$ C contributes to the risk of gastric cancer but not colorectal cancer in the Chinese population: the functional variation of hOGG1 for gastric cancer risk. J Cancer Res Clin Oncol. 2011; 137:1477-1485.

11. Boiteux S and Radicella JP. The human OGG1 gene: structure, functions, and its implication in the process of carcinogenesis. Arch Biochem Biophys. 2000; 377:1-8.

12. Xu X, Wang Y, Guo W, Zhou Y, Lv C, Chen X and Liu $\mathrm{K}$. The significance of the alteration of $8-\mathrm{OHdG}$ in serous ovarian carcinoma. J Ovarian Res. 2013; 6:74.

13. Karihtala P, Kauppila S, Puistola U and Jukkola-Vuorinen A. Absence of the DNA repair enzyme human 8-oxoguanine glycosylase is associated with an aggressive breast cancer phenotype. Br J Cancer. 2012; 106:344-347.

14. Ni J, Mei M and Sun L. Oxidative DNA damage and repair in chronic atrophic gastritis and gastric cancer. Hepatogastroenterology. 2012; 59:671-675.

15. Gao D, Hu J, Zhang X, Gao C and Hong J. Effect of hOGG1 over-expression on cisplatin resistance in esophageal squamous carcinoma cells. Cancer Biother Radiopharm. 2013; 28:433-440.

16. Farinati F, Cardin R, Bortolami M, Nitti D, Basso D, de Bernard M, Cassaro M, Sergio A and Rugge M. Oxidative
DNA damage in gastric cancer: CagA status and OGG1 gene polymorphism. Int J Cancer. 2008; 123:51-55.

17. Canbay E, Agachan B, Gulluoglu M, Isbir T, Balik E, Yamaner S, Bulut T, Cacina C, Eraltan IY, Yilmaz A and Bugra D. Possible associations of APE1 polymorphism with susceptibility and HOGG1 polymorphism with prognosis in gastric cancer. Anticancer Res. 2010; 30:1359-1364.

18. Malik MA, Zargar SA and Mittal B. Lack of influence of DNA repair gene OGG1 codon 326 polymorphisms of gastric cancer risk in the Kashmir valley. Asian Pac J Cancer Prev. 2010; 11:165-168.

19. Weiss JM, Goode EL, Ladiges WC and Ulrich CM. Polymorphic variation in hOGG1 and risk of cancer: a review of the functional and epidemiologic literature. Mol Carcinog. 2005; 42:127-141.

20. Ni M, Qiu J, He W and Wang X. The functional Ser326Cys polymorphism in hOGG1 is associated with gastric cancer risk: evidence from 1180 cases and 2444 controls. Eur J Gastroenterol Hepatol. 2012; 24:683-687.

21. Lu R, Nash HM and Verdine GL. A mammalian DNA repair enzyme that excises oxidatively damaged guanines maps to a locus frequently lost in lung cancer. Curr Biol. 1997; 7:397-407.

22. Yokoyama S, Yamakawa K, Tsuchiya E, Murata M, Sakiyama S and Nakamura Y. Deletion mapping on the short arm of chromosome 3 in squamous cell carcinoma and adenocarcinoma of the lung. Cancer Res. 1992; 52:873-877.

23. Igarashi $M$, Watanabe $M$, Yoshida $M$, Sugaya $K$, Endo Y, Miyajima N, Abe M, Sugano S and Nakae D. Enhancement of lung carcinogenesis initiated with 4-(N-hydroxymethylnitrosamino)-1-(3-pyridyl)-1-butanone by Ogg1 gene deficiency in female, but not male, mice. J Toxicol Sci. 2009; 34:163-174.

24. Tse D, Zhai R, Zhou W, Heist RS, Asomaning K, Su L, Lynch TJ, Wain JC, Christiani DC and Liu G. Polymorphisms of the NER pathway genes, ERCC1 and XPD are associated with esophageal adenocarcinoma risk. Cancer Causes Control. 2008; 19:1077-1083.

25. Dhillon VS, Yeoh E and Fenech M. DNA repair gene polymorphisms and prostate cancer risk in South Australia-results of a pilot study. Urol Oncol. 2011; 29:641-646.

26. Li Z, Guan W, Li MX, Zhong ZY, Qian CY, Yang XQ, Liao L, Li ZP and Wang D. Genetic polymorphism of DNA baseexcision repair genes (APE1, OGG1 and XRCC1) and their correlation with risk of lung cancer in a Chinese population. Arch Med Res. 2011; 42:226-234.

27. Ming-Shiean H, Yu JC, Wang HW, Chen ST, Hsiung CN, Ding SL, Wu PE, Shen CY and Cheng CW. Synergistic effects of polymorphisms in DNA repair genes and endogenous estrogen exposure on female breast cancer risk. Ann Surg Oncol. 2010; 17:760-771.

28. Takezaki T, Gao CM, Wu JZ, Li ZY, Wang JD, Ding JH, Liu YT, Hu X, Xu TL, Tajima K and Sugimura H. hOGG1 Ser(326)Cys polymorphism and modification by 
environmental factors of stomach cancer risk in Chinese. Int J Cancer. 2002; 99:624-627.

29. Gerstenberger JP, Bauer SR, Van Blarigan EL, Sosa E, Song X, Witte JS, Carroll PR and Chan JM. Selenoprotein and antioxidant genes and the risk of high-grade prostate cancer and prostate cancer recurrence. Prostate. 2015; 75:60-69.

30. Figueroa JD, Malats N, Real FX, Silverman D, Kogevinas M, Chanock S, Welch R, Dosemeci M, Tardon A, Serra C, Carrato A, Garcia-Closas R, Castano-Vinyals G, Rothman N and Garcia-Closas M. Genetic variation in the base excision repair pathway and bladder cancer risk. Hum Genet. 2007; 121:233-242.

31. Hu N, Wang Z, Song X, Wei L, Kim BS, Freedman ND, Baek J, Burdette L, Chang J, Chung C, Dawsey SM, Ding T, Gao YT, et al. Genome-wide association study of gastric adenocarcinoma in Asia: a comparison of associations between cardia and non-cardia tumours. Gut. 2015.

32. Shi Y, Hu Z, Wu C, Dai J, Li H, Dong J, Wang M, Miao X, Zhou Y, Lu F, Zhang H, Hu L, Jiang Y, et al. A genomewide association study identifies new susceptibility loci for non-cardia gastric cancer at 3q13.31 and 5p13.1. Nature genetics. 2011; 43:1215-1218.

33. Abnet CC, Freedman ND, Hu N, Wang Z, Yu K, Shu XO, Yuan JM, Zheng W, Dawsey SM, Dong LM, Lee MP, Ding T, Qiao YL, et al. A shared susceptibility locus in PLCE1 at 10q23 for gastric adenocarcinoma and esophageal squamous cell carcinoma. Nature genetics. 2010; 42:764-767.

34. Moser D, Ekawardhani S, Kumsta R, Palmason H, Bock C, Athanassiadou Z, Lesch KP and Meyer J. Functional analysis of a potassium-chloride co-transporter 3 (SLC12A6) promoter polymorphism leading to an additional DNA methylation site. Neuropsychopharmacology. 2009; 34:458-467.

35. Shield AJ, Murray TP, Cappello JY, Coggan M and Board PG. Polymorphisms in the human glutathione transferase Kappa (GSTK1) promoter alter gene expression. Genomics. 2010; 95:299-305.

36. Raptis S, Mrkonjic M, Green RC, Pethe VV, Monga N, Chan YM, Daftary D, Dicks E, Younghusband BH, Parfrey PS, Gallinger SS, McLaughlin JR, Knight JA and Bapat B. MLH1 -93G $>$ A promoter polymorphism and the risk of microsatellite-unstable colorectal cancer. J Natl Cancer Inst. 2007; 99:463-474.

37. French JD, Ghoussaini M, Edwards SL, Meyer KB, Michailidou K, Ahmed S, Khan S, Maranian MJ, O'Reilly M, Hillman KM, Betts JA, Carroll T, Bailey PJ, et al. Functional variants at the $11 \mathrm{q} 13$ risk locus for breast cancer regulate cyclin D1 expression through long-range enhancers. American journal of human genetics. 2013; 92:489-503. 